\title{
INFLUENCIA DE LAS HABILIDADES MATEMÁTICAS BÁSICAS EN EL RENDIMIENTO POSTERIOR
}

\author{
Jessica Mercader Ruiz \\ mercader@uji.es \\ Ma Jesús Presentación Herrero \\ Rebeca Siegenthaler Hierro \\ Universitat Jaume I de Castellón \\ https://doi.org/10.17060/ijodaep.2017.n1.v3.993
}

Fecha de Recepción: 9 Marzo 2017

Fecha de Admisión: 1 Abril 2017

\section{RESUMEN}

El objetivo del presente estudio longitudinal consistió en examinar el poder predictivo de las competencias matemáticas básicas de conteo, operaciones lógicas y habilidades de comparación de magnitudes evaluadas en Educación Infantil sobre los aspectos formales e informales del rendimiento matemático en $2^{\circ}$ de Educación Primaria. La muestra inicial estuvo compuesta por 209 preescolares de 5 a 6 años, de los cuales 180 volvieron a ser evaluados dos años más tarde. En Educación Infantil, se aplicaron diferentes tareas de la batería TEDI-MATH (Grégoire, Noël, y Van Nieuwenhoven, 2005) que evalúan las habilidades de conteo (procedimental y conceptual), operaciones lógicas (seriación, clasificación, conservación e inclusión numérica) y comparación de magnitudes (simbólica y no-simbólica). Dos años más tarde, se administró la batería TEMA-3 (Gingsburg y Baroody, 2003) para evaluar diferentes aspectos relacionados con el rendimiento matemático. Los resultados mostraron que 5 de las 8 tareas aplicadas en Educación Infantil son capaces de predecir el rendimiento matemático posterior, con un especial peso de la habilidad para manejar la secuencia numérica verbal (conteo procedimental). Se comentan las implicaciones de estos hallazgos para la investigación y la práctica psicoeducativa.

Palabras clave: estudio Iongitudinal, rendimiento matemático, conteo, operaciones lógicas, habilidades de comparación.

\section{ABSTRACT}

Influence of early numeracy skills on later performance

The objective of this longitudinal study was to examine the predictive value of early numeracy skills of counting, logical operations and magnitude comparison abilities evaluated in Kindergarten on the formal and informal aspects of mathematical performance in $2^{\text {nd }}$ grade of Primary School. 


\section{INFLUENCIA DE LAS HABILIDADES MATEMÁTICAS BÁSICAS EN EL RENDIMIENTO POSTERIOR}

The initial sample consisted of 209 preschoolers aged 5 to 6 years, 180 of whom were retested two years later. In Kindergarten, different tasks of the TEDI-MATH battery (Grégoire, Noël, \& Van Nieuwenhoven, 2005) were applied, which evaluate counting skills (procedural and conceptual), logical operations (seriation, classification, conservation and inclusion) and magnitudes comparison abilities (symbolic and non-symbolic). Two years later, the TEMA-3 test (Gingsburg \& Baroody, 2003) was administered to assess different aspects related to mathematical performance. The results showed that 5 of the 8 tasks applied in Kindergarten are able to predict the subsequent mathematical performance, with a special weight of the ability to manage verbal numerical sequence (procedural counting). The implications of these findings for psychoeducational research and practice are discussed.

Keywords: Iongitudinal study, mathematical performance, counting, logical operations, comparison skills.

\section{ANTECEDENTES DE LA TEMÁTICA A TRATAR}

La literatura refleja la importancia que una buena competencia matemática tiene para el éxito académico y laboral, así como para la funcionalidad ante tareas de la vida diaria (Geary, 2011). Sin embargo, distintos informes internacionales ponen de manifiesto que existe un elevado porcentaje de sujetos que presentan dificultades en la resolución de tareas matemáticas de carácter básico (Mullis, Martin, Foy, y Hooper, 2016). En virtud de ambos hallazgos, parece plausible continuar profundizando en los factores iniciales que determinan el rendimiento matemático posterior.

En este sentido, tal y como postulan Geary, Hoard, Nugent, y Bailey (2013), las escasas habilidades matemáticas son generalmente consecuencia de una pobre competencia aritmética inicial. Así, la investigación establece que existen determinadas competencias matemáticas básicas ("early numeracy skills") que se constituyen como habilidades iniciales sobre las que se asientan los conocimientos matemáticos de mayor complejidad (Desoete y Grégoire, 2006). Entre ellas, destacan las operaciones lógicas, el conteo, y la comparación de magnitudes, por su carácter informal, su temprana aparición y su influencia en el desarrollo de la cognición matemática, si bien es cierto que los hallazgos acerca de su implicación en el rendimiento matemático posterior y las habilidades que lo componen no son todavía claros.

En lo que se refiere a las operaciones lógicas, diferentes trabajos desde la década de los 70 reflejan que habilidades como la seriación, la clasificación, la conservación o la inclusión propuestas inicialmente por Piaget y Szeminska (1941) son importantes para el rendimiento matemático (Cathcart, 1971; Steffe, 1971). Actualmente, el foco de atención se centra en las habilidades lógicas de los niños más pequeños, dadas las críticas hacia la teoría piagetiana basadas en la importancia que se otorga al estadio evolutivo para el desarrollo de dichas habilidades y, consecuentemente, la infraestimación de su papel en etapas previas del desarrollo (Gelman, Meck, y Merkin, 1986). Los estudios sobre la implicación de las habilidades lógicas tempranas sobre el rendimiento matemático posterior no están exentos de contradicciones. Así, diferentes trabajos apuntan a la importancia de la habilidad temprana de seriación (ordenar un conjunto de acuerdo a sus diferencias numéricas) sobre el rendimiento matemático en los primeros cursos de Educación Primaria (Aunio y Niemivirta, 2010; Grégoire, 2005; Stock, Desoete y Roeyers, 2007; Tobia, Bonifacci, y Marzocchi, 2016). No obstante, otros autores como Stock, Desoete y Roeyers (2009), destacan la relevancia de la habilidad de clasificación (ordenar un conjunto de objetos en función de sus similitudes). El peso de estas habilidades tempranas sobre la habilidad matemática parece extenderse a lo largo de la escolaridad (Desoete, Stock, Schepens, Baeyens, y Roeyers, 2009).

Por otra parte, respecto a las habilidades de iniciales de conteo, diferentes estudios longitudinales ponen de manifiesto la importancia de éstas para el rendimiento matemático en edad escolar 
(Aunio y Niemivirta, 2010; Koponen, Salmi, Eklund, y Aro, 2013; Jordan, Kaplan, Ramineni y Locuniak, 2009). Destacan especialmente los resultados que ofrece un estudio realizado por Aunola, Leskinen, Lerkkanen y Nurmi (2004), los cuales revelan que las habilidades de conteo se alzan como el mejor predictor del rendimiento matemático en Educación Infantil y de las mejoras en éste hasta $2^{0}$ de Educación Primaria. En esta línea, se ha puesto de manifiesto que determinadas habilidades procedimentales de conteo (manejo de la secuencia numérica verbal) en preescolar predicen el rendimiento matemático tanto en Educción Infantil como en Educación Primaria (Östergren y Träff, 2013). Los resultados relativos al conteo conceptual (conocimiento y uso de los principios que rigen el conteo) son más limitados, y están en su mayoría centrados en sujetos con dificultades específicas de aprendizaje (Geary, 2004).

Finalmente, en lo que se refiere a la comparación de magnitudes, esta habilidad (junto con la competencia de subityzing) se establece como una de las competencias que constituyen la habilidad innata de numerosidad (Dehaene, 1997), siendo uno de los aspectos en los que se centra la investigación dadas sus mayores posibilidades a nivel de intervención psicopedagógica. Tradicionalmente, se han utilizado tareas de comparación simbólica (comparación de números arábigos) y no-simbólica (comparación de nubes de puntos) para valorar la habilidad de comparación de magnitudes. No obstante, los hallazgos relativos a su peso sobre el rendimiento matemático posterior no son del todo claros. En este sentido, la revisión de la literatura muestra que el peso de las comparaciones simbólicas o no-simbólicas varía en función del periodo evolutivo en el que se evalúan tanto las habilidades de comparación como el rendimiento matemático posterior. Así, estudios centrados en la etapa preescolar temprana destacan un peso superior de las habilidades de tipo nosimbólico (Libertus, Feigenson, y Halberda, 2013; Mazzocco, Feigenson, y Halberda, 2011). Sin embargo, cuando las habilidades de comparación se evalúan en los últimos cursos de Educación Infantil y el rendimiento matemático se valora en Educación Primaria, parece que es la competencia para la comparación simbólica la que actúa como predictor en este último periodo (De Smedt, Verschaffel, y Ghesquière, 2009; De Smedt, Noël, Gilmore, y Ansari, 2013; Holloway y Ansari, 2009; Sasanguie, Göbel, Moll, Smets, y Reynvoet, 2013; Schneider, et al., 2016).

A modo de conclusión, el conjunto de trabajos revisados pone de manifiesto la necesidad de continuar profundizando en el estudio acerca del peso relativo de las distintas competencias matemáticas básicas sobre el rendimiento matemático posterior desde una perspectiva longitudinal preventiva. Igualmente, el rendimiento matemático se estima, en la mayor parte de los casos, como una medida única que refleja el nivel de competencia del alumno de forma global y abstracta. Por ello, son necesarios más estudios que identifiquen cómo los distintos precursores actúan, no solo ante medidas matemáticas globales, sino también ante habilidades concretas de rendimiento posterior, con la finalidad de programar intervenciones lo más individualizadas y específicas posibles.

\section{OBJETIVOS DE LA INVESTIGACIÓN}

El objetivo del presente trabajo consistió en examinar el poder predictivo de las competencias matemáticas básicas de operaciones lógicas (seriación, clasificación, conservación e inclusión), conteo (procedimental y conceptual) y comparación de magnitudes (simbólica y no-simbólica) evaluadas en Educación Infantil (T1) sobre distintos aspectos formales e informales del rendimiento matemático en $2^{0}$ de Educación Primaria (T2).

\section{MUESTRA Y/O PARTICIPANTES}

La muestra definitiva del estudio estuvo conformada por 180 estudiantes (51.1\% varones; 48.9\% niñas) de 14 centros educativos de las provincias de Castellón y Valencia (España). En el T1, los participantes tenían una edad comprendida entre 5 y 6 años $(M=70.21$ meses; $D T=3.56$ 


\section{INFLUENCIA DE LAS HABILIDADES MATEMÁTICAS BÁSICAS EN EL RENDIMIENTO POSTERIOR}

meses). En el T2, los participantes tenían una edad entre 7 y 8 años $(M=94.16$ meses; $D T=3.78$ meses). Los estudiantes presentaban una media de $\mathrm{Cl}$ equivalente de 99.28 (DT =12.34), que se obtuvo a través de las subpruebas vocabulario y cuadrados de la escala WPPSI (Wechsler, 1981) siguiendo las directrices de Spreen y Strauss (1991). Para la selección de la muestra, se utilizaron como criterios de exclusión la obtención de un $\mathrm{Cl}$ equivalente inferior a 70, así como la presencia en informes escolares de deficiencias sensoriales graves, anomalías neurobiológicas, trastornos psicológicos o deprivación socio-cultural. El 87.8\% de los niños poseían nacionalidad española. Todos los participantes hablaban y comprendían el español. En el T2, el 19.1\% de los participantes asistían a sesiones con especialistas en los respectivos centros escolares: Apoyo Educativo (7.7\%), Educación Compensatoria (1.9\%), Pedagogía Terapéutica (3.3\%), Audición y Lenguaje (3.8\%) y tratamiento combinado (2.4\%).

\section{METODOLOGÍA}

\section{Instrumentos utilizados}

T1-Competencias matemáticas básicas

Para evaluar las competencias matemáticas básicas en Educación Infantil, se aplicaron determinados subpruebas del Test para el diagnóstico de las Competencias Básicas en Matemáticas (TEDI-MATH; Grégoire, et al., 2005). Se trata de un test estandarizado, dirigido a niños de 4 a 8 años, que tiene como finalidad la evaluación de las destrezas matemáticas básicas. Permite, a su vez, identificar sujetos que presenten algún problema en dichas competencias. El test se agrupa en 6 subtest, que a su vez se desglosan en 25 pruebas y distintas subpruebas que se aplican en función del curso y periodo académico. Se seleccionaron tareas relacionadas con las operaciones lógicas (seriación, clasificación, conservación e inclusión) el conteo procedimental y conceptual (contar y numerar) y la comparación de magnitudes simbólica (comparación de números arábigos) y no-simbólica (estimación del tamaño) para evaluar las competencias matemáticas básicas de los participantes. La baremación española del instrumento cuenta con elevados índices de fiabildiad y validez (Grégoire, et al., 2005).

\section{T2-Rendimiento matemático}

Para evaluar el rendimiento matemático en Educación Primaria, se administró el Test de Competencia Matemática Básica (TEMA-3; Gingsburg y Baroody, 2003). Se trata de una prueba estandarizada dirigida a sujetos entre 3 años y 8 años y 11 meses cuyo objetivo es identificar fortalezas y debilidades específicas en la competencia matemática. Se compone de 72 ítems que valoran diferentes aspectos de la competencia matemática infantil. Así, la prueba contempla tanto aspectos informales (aquellos que no requieren el uso de símbolos matemáticos escritos), que son evaluados mediante 81 ítems, como aspectos formales (actividades que implican el uso de símbolos matemáticos), que se engloban en 31 ítems. Todos ellos se recogen en 8 dimensiones agrupadas en siguientes subescalas: a) habilidades informales: numeración, comparación, cálculo y conceptos; b) habilidades formales: convencionalismos, hechos numéricos, caculo y conceptos. La baremación española de la prueba cuenta con elevados índices de fiabilidad y validez (Gingsburg y Baroody, 2003).

\section{Procedimiento}

Tras obtener los permisos de la Consellería de Educación de la Generalitat Valenciana y la aprobación por parte del Comité Ético de la Universitat Jaume I, con la finalidad de abarcar un amplio rango de centros, se seleccionaron 6 estudiantes por aula al azar mediante la técnica de muestreo 
aleatorio simple. La muestra inicial estuvo compuesta por 209 preescolares, de los cuales 180 niños participaron en la investigación en la evaluación en 2 ํㅡㄹ de Educación Primaria (86.6\% de la muestra inicial), conformando así la muestra definitiva del estudio. La evaluación fue llevada a cabo por profesionales del equipo de investigación familiarizados con la aplicación y corrección de los test. La administración de las pruebas se produjo en horario lectivo sin interferir con las actividades significativas del currículum, en espacios cedidos por los centros escolares que reunían las condiciones de ventilación, aislamiento e iluminación óptimas para la evaluación psicopedagógica.

En el T1, se aplicaron las subpruebas seleccionadas del TEDI-MATH (Grégoire, et al., 2005), en una sesión de 30 minutos de duración aproximada. Dos cursos escolares después, se volvió a los colegios donde se administró la prueba estandarizada TEMA-3 (Gingsburg y Baroody, 2003) a los mismos sujetos, de forma individual y en una sesión de 30 minutos.

\section{Análisis estadísticos}

Los análisis estadísticos se realizaron con el software Statistical Package for the Social Science (SPSS), versión 22.00 (SPSS Inc., Chicago, IL USA). Se realizaron distintos análisis de regresión lineal múltiple por el método de pasos sucesivos. Se introdujeron como predictores las puntuaciones directas de ensayos correctos en las subpruebas de contar (conteo procedimental), numerar (conteo conceptual), operaciones lógicas (seriación, clasificación, conservación e inclusión), comparación de números arábigos (comparación simbólica) y estimación del tamaño (comparación nosimbólica) del test TEDI-MATH (Grégoire et al., 2005). Como variables dependientes, se utilizaron las puntuaciones directas en las 8 subescalas del test TEMA-3 (Gingsburg y Baroody, 2003), así como la puntuación total de aciertos.

\section{RESULTADOS ALCANZADOS}

Las competencias matemáticas básicas de conteo procedimental $\left(\Delta R^{2}=.239, p<.001\right)$, seriación $\left(\Delta R^{2}=.057, p<.001\right)$, conservación $\left(\Delta R^{2}=.046, p=.001\right)$ y comparación simbólica $\left(\Delta R^{2}=\right.$ $.039, p=.001)$ predijeron el $38.1 \%$ de la varianza de la puntuación total de rendimiento matemático obtenida en el test TEMA-3 (véase Tabla 1).

Tabla 1. Análisis de regresión de las competencias matemáticas básicas (T1) sobre la $P D$ total de rendimiento matemático (T2).

\begin{tabular}{lllll}
\hline & $\mathrm{F}$ & $R^{2}$ & $\Delta R^{2}$ & $\beta$ \\
\hline TEMA-3: Total rendimiento matemático & & & & \\
Conteo procedimental & & & .239 & .232 \\
Seriación & $26.93^{* *}$ & .381 & .057 & .236 \\
Conservación & & & .046 & .247 \\
Comparación simbólica & & & .039 & .213 \\
\hline
\end{tabular}

Nota: ${ }^{* * * *} p<.001 ; * p<.05$

En lo que respecta a aspectos informales del rendimiento matemático (véase Tabla 2), la habilidad de numeración fue predicha en un $15.2 \%$ por las competencias de conteo procedimental $\left(\Delta R^{2}\right.$ $=.086, p<.001)$, seriación $\left(\Delta R^{2}=.040, p=.005\right)$ y conservación $\left(\Delta R^{2}=.025, p=.023\right)$. En el caso de la variable comparación, las habilidades iniciales de seriación $\left(\Delta R^{2}=.174, p<.001\right)$, comparación simbólica $\left(\Delta R^{2}=.052, p=.001\right)$ y conservación $\left(\Delta R^{2}=.028, p=.011\right)$ resultaron predictores significativos, explicando en su conjunto el $25.4 \%$ de la varianza. Las habilidades de conteo proce- 


\section{INFLUENCIA DE LAS HABILIDADES MATEMÁTICAS BÁSICAS EN EL RENDIMIENTO POSTERIOR}

dimental $\left(\Delta R^{2}=.158, p<.001\right)$, seriación $\left(\Delta R^{2}=.054, p=.001\right)$, conservación $\left(\Delta R^{2}=.029, p=\right.$ .010) y comparación simbólica $\left(\Delta R^{2}=.021, p=.028\right)$ predijeron el $24.6 \%$ de la varianza en tareas de cálculo informal. Por último, las competencias iniciales de comparación simbólica $\left(\Delta R^{2}=.115\right.$, $p<.001)$, conservación $\left(\Delta R^{2}=.071, p<.001\right)$, seriación $\left(\Delta R^{2}=.034, p=.006\right)$ e inclusión $\left(\Delta R^{2}\right.$ $=.151, p=.021)$ predijeron el $24.2 \%$ del conocimiento informal de conceptos.

Tabla 2. Análisis de regresión de las competencias matemáticas básicas (T1) sobre las habilidades informales de rendimiento matemático (T2).

\begin{tabular}{|c|c|c|c|c|}
\hline & F & $R^{2}$ & $\Delta R^{2}$ & $\beta$ \\
\hline \multicolumn{5}{|c|}{ TEMA-3: Habilidades informales } \\
\hline \multicolumn{5}{|l|}{ Numeración } \\
\hline Conteo procedimental & \multirow{3}{*}{$10.49^{* *}$} & \multirow{3}{*}{.152} & .086 & .155 \\
\hline Seriación & & & .040 & .147 \\
\hline Conservación & & & .025 & .175 \\
\hline \multicolumn{5}{|l|}{ Comparación } \\
\hline Seriación & \multirow{3}{*}{$20.01 * *$} & \multirow{3}{*}{.254} & .174 & .347 \\
\hline Comparación simbólica & & & .052 & .228 \\
\hline Conservación & & & .028 & .171 \\
\hline \multicolumn{5}{|l|}{ Cálculo } \\
\hline Conteo procedimental & \multirow{4}{*}{$15.61 * *$} & \multirow{4}{*}{.246} & .158 & .180 \\
\hline Seriación & & & .054 & .236 \\
\hline Conservación & & & .029 & .196 \\
\hline Comparación simbólica & & & .021 & .155 \\
\hline \multicolumn{5}{|l|}{ Conceptos } \\
\hline Comparación simbólica & \multirow{4}{*}{$13.95 * *$} & \multirow{4}{*}{.242} & .115 & .233 \\
\hline Conservación & & & .071 & .229 \\
\hline Seriación & & & .034 & .169 \\
\hline Inclusión & & & .021 & .164 \\
\hline
\end{tabular}

Nota: $* * p<.001 ; * p<.05$

En el caso de las habilidades formales (véase Tabla 3), el manejo de convencionalismos fue predicho por las competencias básicas de conteo procedimental $\left(\Delta R^{2}=.128, p<.001\right)$ y seriación $\left(\Delta R^{2}=.040, p=004\right)$, explicando en su conjunto el $16.8 \%$ de la varianza. Las competencias básicas de conteo procedimental $\left(\Delta R^{2}=.196, p<.001\right)$, conservación $\left(\Delta R^{2}=.055, p<.001\right)$, comparación simbólica $\left(\Delta R^{2}=.039, p=.002\right)$ y seriación $\left(\Delta R^{2}=.151, p<.001\right)$ explicaron el $29.5 \%$ de la varianza de la habilidad de recuperación de hechos numéricos. Estas mismas variables predijeron la ejecución ante tareas de cálculo formal en un $43 \%$ (conteo procedimental, $\Delta R^{2}=.284, p<.00$; seriación, $\Delta R^{2}=.056, p<.001$; conservación, $\Delta R^{2}=.046, p<.001$; comparación simbólica, $\Delta R^{2}$ $=.043, p<.001)$. Un $28.7 \%$ de la varianza del conocimiento de conceptos de carácter formal fue predicho por las competencias de conteo procedimental $\left(\Delta R^{2}=.213, p<.001\right)$, conservación $\left(\Delta R^{2}\right.$ $=.052, p<.001)$ y seriación $\left(\Delta R^{2}=.022, p=.021\right)$. 
Tabla 3. Análisis de regresión de las competencias matemáticas básicas (T1) sobre las habilidades formales de rendimiento matemático (T2).

\begin{tabular}{lllll}
\hline & $\mathrm{F}$ & $R^{2}$ & $\Delta R^{2}$ & $\beta$ \\
\hline TEMA-3: Habilidades formales & & & & \\
Convencionalismos & & & & \\
$\quad$ Conteo procedimental & $17.83 * *$ & .168 & .128 & .268 \\
$\quad$ Seriación & & & .040 & .219 \\
Hechos numéricos & & & .179 & .196 \\
$\quad$ Conteo procedimental & $18.30 * *$ & .295 & .055 & .252 \\
$\quad$ Conservación & & & .039 & .213 \\
$\quad$ Comparación simbólica & & & .021 & .160 \\
$\quad$ Seriación & & & .284 & .272 \\
Cálculo & $33.02 * *$ & .430 & .056 & .236 \\
$\quad$ Conteo procedimental & & & .046 & .248 \\
$\quad$ Seriación & & & .043 & .224 \\
Conservación & & & .213 & .318 \\
$\quad$ Comparación simbólica & & & & \\
Conceptos & $23.62 * *$ & .287 & .052 & .227 \\
$\quad$ Comparación simbólica & & & .022 & .163 \\
$\quad$ Conservación & & & & \\
$\quad$ Seriación & & & & \\
\hline
\end{tabular}

Nota: ${ }^{* *} p<.001 ; * p<.05$

\section{DISCUSIÓN}

El objetivo del presente trabajo consistió en analizar el poder predictivo que poseen las competencias matemáticas básicas de operaciones lógicas (seriación, clasificación, conservación e inclusión), conteo (procedimental y conceptual) y habilidades de comparación (simbólica y no-simbólica) en Educación Infantil sobre el rendimiento matemático en 2ํㅜ de Educación Primaria, evaluado a través de una medida global que se desglosa en diferentes competencias específicas.

En lo que se refiere al rendimiento matemático global, el conjunto de competencias matemáticas básicas analizadas explicaron el $38.1 \%$ de la varianza. Concretamente, el manejo de la secuencia numérica verbal fue el predictor con más peso, en la línea de otros estudios longitudinales que destacan la implicación de las habilidades tempranas de conteo en el rendimiento matemático posterior (Aunola et al., 2004; Aunio y Niemivirta, 2010; Jordan at al., 2009; Koponen, et al., 2013), y, especialmente, de hallazgos que sugieren la especial relevancia de las habilidades procedimentales (Östergren y Träff, 2013). También, las habilidades lógicas de seriación, la capacidad de conservación numérica y la habilidad de comparación de números arábigos, ofrecieron una contribución significativa adicional a la explicación del rendimiento matemático global en $2^{\circ}$ de Educación Primaria en la línea de otros estudios previos (Aunio y Niemvirta, 2010; De Smedt et al., 2009; De Smedt et al., 2013; Grégoire, 2005; Holloway y Ansari, 2009; Schneider et al., 2016; Sasanguie et al., 2013; Tobia et al., 2016; Stock et al., 2007; Stock et al., 2009).

Respecto a las distintas habilidades matemáticas específicas analizadas en $2^{\circ}$ de Educación Primaria, el peso de las competencias matemáticas básicas fue superior en el caso de las tareas de tipo formal, las cuales requieren el uso de los símbolos matemáticos escritos. En la línea de la afirmación propuesta por Geary et al. (2013), las escasas habilidades matemáticas en la edad escolar podrían ser explicadas, en parte, en base a una pobre competencia inicial. 


\section{INFLUENCIA DE LAS HABILIDADES MATEMÁTICAS BÁSICAS EN EL RENDIMIENTO POSTERIOR}

Específicamente, destaca el poder de predicción de la habilidad para enumerar verbalmente el conteo, dado su peso superior en distintas habilidades específicas de tipo informal (numeración y cálculo), formal (lectura y escritura de números, recuperación de hechos numéricos y cálculo). La habilidad lógica de seriación, por su parte, fue especialmente importante para la habilidad informal que requiere el establecimiento de relaciones de distancia entre los números (comparación). La habilidad de comparación de números arábigos parece ser la que tiene mayor peso en el caso del conocimiento y uso de conceptos. Cabe resaltar que las habilidades lógicas de conservación y, en menor medida, de inclusión ofrecieron una contribución añadida en algunas de las habilidades específicas analizadas. El conjunto de estos resultados sugieren que la influencia de una u otra competencia matemática básica variaría en función del tipo de tarea matemática que se presente y de su carácter (formal o informal).

Finalmente, la habilidad lógica de clasificación, el conocimiento de los conceptos que rigen los principios del conteo, así como las habilidades de comparación de tipo no-simbólico, no actuaron como predictores significativos en ninguno de los casos. La ausencia de resultados significativos respecto a la tarea de clasificación podría relacionarse, como se ha comentado anteriormente, con una especial dificultad que los sujetos de la presente muestra mostraron para realizar dicha tarea. En lo que se refiere al conteo conceptual, en la línea de la propuesta de Geary (2004), el conocimiento conceptual podría estar a la base de dichas habilidades procedimentales, pudiendo actuar a través de las mismas. Respecto a las habilidades de comparación no-simbólica, parece que el peso sobre el de dichas habilidades rendimiento general se manifestaría en etapas previas a las que se recogen en el presente estudio (Libertus et al., 2013a; Libertus et al., 2013b; Mazzocco et al., 2011). Tal y como sugieren Holloway y Ansari (2009) los resultados obtenidos parecen corroborar que, en el periodo de edad analizado, la relación entre habilidades tempranas de comparación y rendimiento matemático posterior tendría un carácter indirecto, mediado por la experiencia con los símbolos numéricos.

El presente trabajo no está exento de limitaciones. Futuras investigaciones deberían contar con investigaciones longitudinales más extensas en el tiempo, con el objetivo de determinar cómo los diferentes predictores actúan en función del periodo evolutivo. También, en lo referente a los instrumentos de evaluación, sería interesante que futuros estudios se sirvieran de escalas de estimación con el objetivo de otorgar validez ecológica a las estimaciones del rendimiento matemático. Finalmente, sería interesante incorporar, junto con las competencias matemáticas básicas, otras variables cuya relación con el rendimiento matemático ha sido demostrada (e.g. funcionamiento ejecutivo, motivación, variables contextuales).

Por último, del presente estudio se desprenden implicaciones para la investigación y la práctica psicoeducativa. Respecto a la investigación, cabe destacar la importancia de incorporar modelos multi-factoriales que integren tanto diversas competencias matemáticas básicas como diferentes medidas de resultados matemáticos posteriores en la explicación del aprendizaje matemático a partir de los 5 años. En lo que se refiere a la práctica, se resalta la importancia de incorporar, desde Educación Infantil, los aspectos mencionados en términos de intervención, con el objetivo de optimizar el desarrollo matemático desde una perspectiva integral y preventiva y reducir así el impacto hacia posibles dificultades que puedan producirse en etapas posteriores del desarrollo. En este sentido, se resalta la existencia de algunos programas de evidencia empírica demostrada que trabajan competencias matemáticas específicas en la etapa preescolar (e.g. Big Math for Little Kids; Ginsburg, Greenes, y Balfanz, 2003). Estos programas, están basados en lo que los niños conocen y en aquello que son capaces de hacer, integrando las distintas habilidades en actividades motivadoras en el contexto del aula en base a los intereses específicos de los niños. 


\section{CONCLUSIONES}

Los resultados del presente estudio muestran que existen determinadas competencias matemáticas básicas en Educación Infantil que son importantes para el rendimiento posterior. Destaca especialmente el peso de la habilidad para enunciar y manejar la secuencia numérica verbal. Igualmente, se observa que la influencia de una u otra competencia matemática básica varía en función del tipo de tarea matemática que se presente en etapas posteriores.

\section{FINANCIACIÓN:}

El presente trabajo ha sido financiado por el Ministerio de Economía y Competitividad de España (EDU2012-37452) y la Universitat Jaume I de Castellón (beca pre-doctoral; 2I005-PREDOC/2013/34).

\section{REFERENCIAS BIBLIOGRÁFICAS}

Aunio, P., y Niemivirta, M. (2010). Predicting children's mathematical performance in grade one by early numeracy skills. Learning and Individual Differences, 20, 427-435.

Aunola, K., Leskinen, E., Lerkkanen, M.K., y Nurmi, J.E. (2004). Developmental dynamics of math performance from preschool to grade 2. Journal of Educational Psychology, 96(4), 699-713.

Cathcart, W.G. (1971). The relationship between primary students' rationalization of conservation and their mathematical achievement. Child Development, 42(3), 755-765.

De Smedt, B., Noël, M. P., Gilmore, C., y Ansari, D. (2013). How do symbolic and non-symbolic numerical magnitude processing skills relate to individual differences in children's mathematical skills? A review of evidence from brain and behavior. Trends in Neuroscience and Education, 2(2), 48-55.

De Smedt, B., Verschaffel, L., y Ghesquière, P. (2009). The predictive value of numerical magnitude comparison for individual differences in mathematics achievement. Journal of Experimental Child Psychology, 103(4), 469-479.

Dehaene, S. (1997). The number sense: How the mind creates mathematics. New York (NY): Oxford University Press.

Desoete, A., Stock, P., Schepens, A., Baeyens, D., y Roeyers, H. (2009). Classification, seriation, and counting in grades 1, 2, and 3 as two-year longitudinal predictors for low achieving in numerical facility and arithmetical achievement? Journal of Psychoeducational Assessment, 27(3), 252-264.

Desoete, A., y Grégoire, J. (2006). Numerical competence in young children and in children with mathematics learning disabilities. Learning and Individual Differences, 16(4), 351-367.

Geary, D. C. (2004). Mathematics and learning disabilities. Journal of learning disabilities, 37(1), 415.

Geary, D.C. (2011). Consequences, characteristics, and causes of mathematical learning disabilities and persistent low achievement in mathematics. Journal of Developmental and Behavioral Pediatrics: JDBP, 32(3), 250-263.

Geary, D.C., Hoard, M.K., Nugent, L., y Bailey, D.H. (2013). Adolescents' functional numeracy is predicted by their school entry number system knowledge. PloS one, 8(1), e54651.

Gelman, R., Meck, E., y Merkin, S. (1986). Young children's numerical competence. Cognitive Development, 1, 1-29.

Ginsburg, H. y Baroody, A. (2003). TEMA-3; Test de Competencia Matemática Básica. Madrid: TEA Ediciones.

Ginsburg, H.P., Greenes, C., y Balfanz, R. (2003). Big Math for Little Kids. Parsippany, NJ: Dale Seymour Publications. 


\section{INFLUENCIA DE LAS HABILIDADES MATEMÁTICAS BÁSICAS EN EL RENDIMIENTO POSTERIOR}

Grégoire, J. (2005). Logical development and arithmetic competency: Is the Piagetan model always current? En Crahay, M., Verschaffel, L., y Grégoire, J. (Eds.), Enseignement et appretissage des mathématiques (pp. 57-77). Bruxelles, Bélgice: De Boeck.

Grégoire, J., Nöel, M., y Van Nieuwenhoven, C. (2005). TEDI-MATH; Test para el Diagnostico de las Competencias Básicas en Matemáticas. Madrid: TEA Ediciones.

Holloway, I. D., y Ansari, D. (2009). Mapping numerical magnitudes onto symbols: The numerical distance effect and individual differences in children's mathematics achievement. Journal of Experimental Child Psychology, 103(1), 17-29.

IBM Corp. Released (2013). IBM SPSS Statistics for Windows, Version 22.0. Armonk, NY: IBM Corp.

Jordan, N.C., Kaplan, D., Ramineni, C., y Locuniak, M.N. (2009). Early math matters: kindergarten number competence and later mathematics outcomes. Developmental Psychology, 45(3), 850867.

Koponen, T., Salmi, P., Eklund, K., y Aro, T. (2013). Counting and RAN: Predictors of arithmetic calculation and reading fluency. Journal of Educational Psychology, 105(1), 162-175.

Libertus, M.E., Feigenson, L., y Halberda, J. (2013). Numerical approximation abilities correlate with and predict informal but not formal mathematics abilities. Journal of Experimental Child Psychology, 116(4), 829-838.

Mazzocco, M.M., Feigenson, L., y Halberda, J. (2011). Preschoolers' precision of the approximate number system predicts later school mathematics performance. PLoS one, 6(9), e23749.

Mullis, I.V.S., Martin, M.O., Foy, P., y Hooper, M. (2016). TIMSS 2015 International Results in Mathematics. Recuperado de: http://timssandpirls.bc.edu/timss2015/ international-results/

Östergren, R., y Träff, U. (2013). Early number knowledge and cognitive ability affect early arithmetic ability. Journal of Experimental Child Psychology, 115(3), 405-421.

Piaget, J., y Szeminska, A. (1941). La genèse du nombre chez l'enfant. Neuchâtel: Delanchaux et Niestlé.

Sasanguie, D., Göbel, S.M., Moll, K., Smets, K., y Reynvoet, B. (2013). Approximate number sense, symbolic number processing, or number-space mappings: What underlies mathematics achievement? Journal of Experimental Child Psychology, 114(3), 418-431.

Schneider, M., Beeres, K., Coban, L., Merz, S., Schmidt, S., Stricker, J., y De Smedt, B. (2016). Associations of non-symbolic and symbolic numerical magnitude processing with mathematical competence: a meta-analysis. Developmental Science, 1-16. doi: 10.1111/desc.12372

Spreen, 0., y Strauss, E. (1991). A compendium of neuropsychological tests: administration, norms, and commentary. New York: Oxford University Press.

Steffe, L. (1971). The realtionship between conservation of numerousness to problem solving ability in first grade children. Journal of Research in Mathematics Education, 15(1), 47-52.

Stock, P., Desoete, A., y Roeyers, H. (2007). Early markers for arithmetic difficulties. Educational and Child Psychology, 24, 28-39.

Stock, P., Desoete, A., y Roeyers, H. (2009). Predicting arithmetic abilities: The role of preparatory arithmetic markers and intelligence. Journal of Psychoeducational Assessment, 27(3), 237-251.

Tobia, V., Bonifacci, P., y Marzocchi, G.M. (2016). Concurrent and longitudinal predictors of calculation skills in preschoolers. European Journal of Psychology of Education, 31(2), 155-174.

Wechsler, D. (1981). Escala de Inteligencia de Wechsler para Preescolar y Primaria. Madrid: TEA Ediciones. 